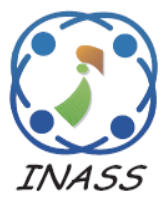

http://www.inass.org/

\title{
Alcohol Expectancy Prediction Using Fuzzy C-Regression Based Structural Brain Imaging
}

\author{
Gayathri Selvaraj $^{1 *}$ \\ Punithavalli Muthuswamy ${ }^{1}$ \\ ${ }^{1}$ Department of Computer Applications, Bharathiar University, Coimbatore, India \\ *Corresponding author's Email: gayathrisphd123@gmail.com
}

Chaitanya Vasanth Kumar ${ }^{1}$

\begin{abstract}
An Alcohol Use Disorder (AUD) can be easily predicted by using General Linear Model (GLM) which evaluates the interaction of alcohol expectancy and impulsivity associated with the reduced Gray Matter Volume (GMV) of the right posterior insula in women and the left thalamus in both women and men. However, this analysis does not support the detection of non-linear distribution of GMV at the human brain. Therefore, in this paper, detection of GMV reduction of a brain also known as Alcoholism Detection (AD) is improved by using a Fuzzy CRegression (FCR) model that supports the non-linearity of gray matter in the brain. In this method, spatial information is also incorporated into the FCR model to remove the noise in the brain images. Moreover, the Alcohol Expectancy Test (AET) score is estimated based on the deviations of GMV that mediates the correlation between GMV and alcoholism. This AET score is mostly related to the reduced GMV of the left thalamus in women and men combined and in men alone. Thus, the GMV reduction in the right posterior insula and left thalamus indicates the relationship between AET score and alcoholism. Finally, the experimental outcomes demonstrate that the proposed FCRAD method achieves an accuracy of $86.06 \%$ which is $8.77 \%$ higher than the existing GLMAD method.
\end{abstract}

Keywords: Alcoholism, Data mining, Gray matter volume, General linear model, Fuzzy c-regression.

\section{Introduction}

Generally, alcoholism or alcohol addiction is referred to as a human disease or a chronic disease characterized by a lack of ability to control drinking or absorption level of alcohol. It causes several long-term and short-term consequences in which the short-term consequences have more hazardous than the long-term consequences [1]. The short-term consequences contain breathing trouble, shrivel intellect actions, distorted visualization, worse selfconsciousness, etc. The long-term consequences may include brain defects, liver diseases, heart problems, bone loss, birth defects, increased risk of cancer, etc. There are different reliable tests available for the actual utilization of alcohol whereas the most common test is known as Blood Alcohol Content (BAC). This test is mostly used for verifying the alcohol tolerance. The severe injuries of alcoholism in the human brain are predicted based on the utilization of data mining algorithms since it provides a better decision and diagnosis. Normally, data mining algorithms can deal with the problem of how best to use this data for discovering new knowledge and improving the decision-making process. As a result, those algorithms are applied in medical sciences to offer an accurate diagnosis of the patient disease based on the consideration of the crucial features from a large amount of data [2]. Those features are extracted by using learning processes like regression, clustering, association rule mining and classification.

In the past decades, structural brain imaging was developed, namely GLM-based AD (GLMAD) [3] to examine the neural bases underlying the relationship between the risk factors and problem drinking. The risk factors were alcohol expectancy and impulsivity which are estimated by the GLM. The alcohol expectancy was allied with the reduced GMV of the right posterior insula in women and the correlation of alcohol expectancy and impulsivity were related to the reduced GMV of the left 
thalamus in both women and men. Also, a VoxelBased Morphometry (VBM) analysis was performed for predicting the structural brain correlates of these risk variables. However, this analysis may support only the linear distribution of GMV whereas the non-linear distribution of GMV was not detected efficiently.

Hence in this article, GMV segmentation is improved by using an FCR model that supports the non-linearity of gray matter in the brain. In this method, spatial information is also incorporated into the FCR model to remove the noise in the brain images and segment the brain image into graymatter images that derive the Regions-Of-Interest (ROI) i.e., the right posterior insula in women and the left thalamus in men and women. Then, mediation analyses are performed to examine the relationship between GMV correlates of alcoholism and AET score. This model reduces the complexity of segmentation of GMV and detects the GMV reduction in insula and thalamus regions of both men and women.

The rest of the article is structured as follows: Section 2 presents the literature survey related to the brain image segmentation and classification. Section 3 describes the proposed methodology. Section 4 illustrates the experimental results of the proposed approach. Finally, Section 5 concludes the research work and presents future enhancement.

\section{Literature survey}

A semi-supervised clustering technique [4] was proposed to segment the brain image into different non-overlapping homogeneous regions by considering the image pixel intensity values as features and identify the region containing the tumor. This approach was applied to retrieve the segmentation of normal MR brain images and MR brain images with multiple sclerosis lesions projected on z planes. In this technique, three cluster validity indices were used as the objective functions and optimized by using a modern multiobjective optimization technique named AMOSA according to the simulated annealing concepts. The first two cluster validity indices were symmetry distance based Sym-index and Euclidean distance based Iindex along with the unsupervised properties. The third cluster validity index was Minkowski index. However, it uses same number of sub-clusters per cluster and requires some amount of labeled data as the supervised information which was complex for few cases.

A Back Propagation Neural Network (BPNN) based technique [5] was proposed to classify the given MR brain image as normal structure (nonoccurrences of diseases) or abnormal structure (occurrences of diseases such as glioma, Alzheimer, meningioma, Alzheimer plus visual agnosia, Pick's disease, sarcoma, Huntington's disease). At first, feature extraction was performed by using wavelet transform and the features dimensionality was reduced based on the Principle Component Analysis (PCA). Once the features were extracted, BPNN was adopted with Scaled Conjugate Gradient (SCG) to discover the optimal weights of the NN. On the other hand, it requires noise removal process to remove the noisy features and also the feature extraction was time-consuming.

An automated diagnosis [6] was proposed based on the classification of MR brain images as whether the image was that of a normal brain or a pathological brain i.e., suffering from brain lesion. In this technique, two processes were performed such as feature extraction and classification. Initially, the features from MR brain images were obtained by using Discrete Wavelet Transformation (DWT). The extracted features were reduced by using PCA for obtaining more significant features. Then, Feed Forward back propagation Artificial Neural Network (FP-ANN) and KNN classifiers were applied for the classification process. Nonetheless, this technique was sensitive to noisy or irrelevant features.

A decision tree based classification [7] was proposed to classify the brain tumor by using MR brain images. In this technique, four processes were performed such as pre-processing, feature extraction, association rule mining and classification. The median filtering process was used in pre-processing to enhance the brain image and the features were extracted by using the texture feature extraction method. After that, the association rules were mined by using the extracted features. Finally, the Naive Bayes (NB) and Decision Tree (DT) were applied to classify the brain tumor based on the association rules. Conversely, this technique was only suitable for large datasets i.e., an accurate outcome was provided only with more number of data/attributes. If the amount of data was reduced, then the accuracy of this method was also decreased gradually.

Brain tumor classification [8] was proposed by using Discrete Cosine Transformation (DCT) with Probabilistic NN (PNN). In this technique, DCT was used for extracting the features and reducing their dimensionality. The PNN was used for classifying the brain tumor and providing a better diagnosis. However, PNN was slower than multilayer perceptron networks at classifying new cases and 
not accurately eliminates the noisy features from the brain tumor images.

An MRI based brain tumor feature extraction and detection [9] were proposed based on the biologically inspired Berkeley Wavelet Transformation (BWT) and Support Vector Machine (SVM). In this technique, BWT based brain tumor segmentation was used for segmenting the tumor regions from medical images. From the segmented tumor region, the relevant features were extracted. Then, the extracted features were classified as healthy or infected tumor tissues by using the SVM based classifier. However, the classification accuracy was still less and the complexity of this method was high since it requires extensive memory for classification.

Children brain tumor classification [10] was proposed based on data mining techniques. Initially, the noise from the MR brain images was removed by a median filter. Then, ROI i.e., tumor region was extracted and the features related with the brain images were obtained by using Haar Wavelet Transform (HWT). Finally, Tree-Augmented Bayesian Networks Nearest Neighbor (TANNN) classifier was applied to detect the type of brain tumors that affect the children. However, it fails to cluster the noisy features and not suitable for nonlinear datasets i.e., the dataset which contains nonlinearly organized data.

\subsection{Overview of literature survey}

\section{i) K-NNAD \& ANNAD method}

In the KNNAD method [6], consider the training dataset $D=\left\{y_{i}, c_{i}\right\}, i=1, \ldots, n$ where $y_{i}$ is a training sample, $c_{i}$ is its corresponding class and $n$ is the number of training samples. Then, the distances between the test samples and all the training samples are computed. The $k$-nearest neighbors are selected to vote for the class of the new sample. The $k$ value is tuned until a reasonable level of correctness is achieved. Therefore, the proper $k$ value should be chosen to achieve the highest accuracy.

In the ANNAD method [6], the neural network is trained for fine-tuning the weights and biases in order to achieve the desired mapping. The image samples are given as input to the network. Then, the variable parameters, weights and biases of the network are adjusted by the network itself to capture the relationship between the input and output i.e., GMV reduction is detected.

ANN consists of 3 layers, namely input, hidden and output layer. The image samples $x_{i}, i=1, \ldots, n$ are given to the input layer. The hidden layer of ANN is defined as tan-sigmoid transfer function.

$$
f\left(x_{i}\right)=\frac{2}{1+e^{-2 x_{i}}}-1
$$

Each input image sample has its own weight values as $w_{1}, w_{2}, \ldots, w_{n}$ and the weighted sum of the inputs is done by the adder function as follows:

$$
u=\sum_{i=1}^{n} w_{i} x_{i}
$$

The output layer of ANN is described by the following equation.

$$
y=f\left(\sum_{i=1}^{n} w_{i} x_{i}+b_{i}\right)
$$

In the Eq. (3), $y$ is the output neuron value; $f(x)$ is the transfer function, $w_{i}$ refers the weight values, $x_{i}$ denotes input data values and $b_{i}$ refers to the bias value. Based on the output neuron values, variations in the GMV reduction are detected. However, it requires high processing time if neural network is large. Also, it is difficult to know how many neurons and layers are needed.

\section{ii) NBAD \& DTAD method}

In the NBAD method [7], the conditional probability that an image $x$ belongs to a class $k$ can be computed from the conditional probabilities of finding specific images in each class and the unconditional probability of the images in each class. For a given image $x \in X$ and $c$ classes, where $X$ is the random variable. The conditional probability that an image $x$ belongs to a class $k$ can be computed as follows:

$$
P\left(c_{k} \mid x\right)=P\left(c_{k}\right) \frac{P\left(x \mid c_{k}\right)}{P(x)}
$$

Eq. (4) indicates that the computation of $P\left(c_{k} \mid x\right)$ is a detection problem since it finds the probability that the given image $x$ belongs to class $k$ and the optimum class is decided by selecting the class with the highest probability among all possible classes which can reduce the prediction error. Consider that any specific value of image $x$ conditional on $c_{k}$ is statistically independent of each dimension and as follows:

$$
P\left(x \mid c_{k}\right)=\prod_{i=0}^{n} P\left(x_{i} \mid c_{k}\right)
$$

Here, $n$ is the number of images. By combining the Eq. (4) and (5), the NB classifier is defined as: 


$$
k=\arg \max _{k} P\left(c_{k}\right) \prod_{i=0}^{n} P\left(x_{i} \mid c_{k}\right)
$$

The value of $P(x)$ is neglected since the value is similar for all class. Thus, the alcoholism is detected; however, it requires more data to provide the highest accuracy.

In the DTAD method [7], the expected information from a training dataset $X$ with $k$ number of classes $C$ and a total of $|X|$ cases is given as:

$$
I(X)=-\sum_{j=1}^{k} \frac{f\left(C_{j}, X\right)}{|X|} \log _{2}\left(\frac{f\left(C_{j}, X\right)}{|X|}\right)
$$

Here, $\frac{f\left(C_{j}, X\right)}{|X|}$ is the probability of occurrence of class $C_{j}$. If the training set is split according to the response variable $h$, there may be $n$ number of cases. Then, the expected information is defined as the weighted sum over the subsets.

$$
I_{S}(X)=-\sum_{i=1}^{n} \frac{\left|X_{i}\right|}{|X|} I\left(X_{i}\right)
$$

By splitting the training dataset, the information gain is defined as:

$$
G(X)=I(X)-I_{S}(X)
$$

Also, the gain ratio is defined as follows:

$$
\begin{aligned}
& \text { Gain Ratio }(G R)=G(X) / \operatorname{Split} \operatorname{Info}(X) \\
& \text { Split } \operatorname{Info}(X)=-\sum_{i=1}^{n} \frac{\left|X_{i}\right|}{|X|} \log _{2}\left(\frac{\left|X_{i}\right|}{|X|}\right)
\end{aligned}
$$

Gain ratio is the fraction of useful data for prediction generated due to the split. The experiment which maximizes the ratio, subject to the constraint of large information gain is chosen. Training images (root node) are split and the branches are generated by the following criteria. At some node, sample distribution in each group becomes uniform and so the information is largest. These are defined as leaf nodes i.e., true classes. This process is continued until all the images belong to the same class or all the images have identical attribute values. However, the computation complexity is high while various values are unclear or various outcomes are correlated.

\section{iii) SVMAD method}

In the SVMAD method [9], consider the training dataset as a set of instance-label pairs $\left(x_{i}, y_{i}\right), i=$ $1, \ldots, n, x_{i} \in \mathbb{R}^{n}, y_{i} \in\{-1,+1\}$ where $x_{i}$ denotes the instances and $y_{i}$ denotes the labels. The optimal hyperplane with the maximal margin is achieved by solving the following unconstrained optimization problem for different classes:

$$
\min _{w} \frac{1}{2} w^{T} w+T \sum_{i=1}^{n} \xi\left(w ; x_{i}, y_{i}\right)
$$

In Eq. (12), $T>0$ denotes the penalty parameter and $w$ denotes the weight of training images $x_{i}$. By solving this optimization problem, alcoholism i.e., GMV reduction is detected. However, the training time is high.

\section{iv) GLMAD method}

Apart from those above discussed methods, the GLMAD method [3] uses three regressions for ROI segmentation as:

$$
\begin{aligned}
& Y=i_{1}+c X+e_{1} \\
& Y=i_{2}+c^{\prime} X+b M+e_{2} \\
& M=i_{3}+a X+e_{3}
\end{aligned}
$$

Where $a$ denotes $X \rightarrow M, b$ denotes $M \rightarrow Y, c^{\prime}$ represents $X \rightarrow Y$ and $c$ represents $X \rightarrow Y$. The constants $i_{1}, i_{2}, i_{3}$ are the intercepts and $e_{1}, e_{2}, e_{3}$ are the residual errors. Also, Fuzzy C-Means (FCM) based clustering was applied in which Euclidean distance between the cluster center and the data sample was used for clustering the identical regressions to identify the reduced GMV. The objective function of the FCM is to minimize:

$$
\begin{aligned}
& J(U, V)=\sum_{k=1}^{N} \sum_{i=1}^{c}\left(\mu_{i k}^{m}\right) E_{i k}^{2} \\
& \text { Euclidean distance, } E_{i k}^{2}=\left\|x_{k}-v_{i}\right\|^{2}
\end{aligned}
$$

Here, $x_{k}$ denotes $k^{t h}$ data point, $v_{i}$ denotes $i^{t h}$ cluster center and $U$ denotes the fuzzy membership matrix. The steps for FCM are:

Consider $X=\left\{x_{1}, x_{2}, \ldots, x_{N}\right\}$ is the set of data points and $V=\left\{v_{1}, v_{2}, \ldots, v_{c}\right\}$ is the set of cluster centers.

Step 1: Randomly choose $c$ cluster centers.

Step 2: Compute the fuzzy membership $\mu_{i k}$ as:

$$
\mu_{i k}=1 / \sum_{k=1}^{c}\left(\frac{E_{i k}}{E_{j k}}\right)^{2 /(m-1)}
$$

Step 3: Compute the fuzzy centers $v_{i}$ as: 


$$
\underset{1, \ldots, c}{v_{i}=\left(\sum_{k=1}^{N}\left(\mu_{i k}\right)^{m} x_{k}\right) /\left(\sum_{k=1}^{N}\left(\mu_{i k}\right)^{m}\right), \forall i=}
$$

Step 4: Repeat Step $2 \& 3$ until the minimum $J$ value is achieved.

However, this approach was very sensitive to the noise in the medical images and Euclidean distance may measure unequally weight underlying factors. Also, it can be either hyper-spherical or hyperellipsoidal; thus, it may not provide accurate results in situations where data has arbitrary shapes. As a result, FCR-based clustering is proposed in this research work to obtain accurate detection of alcoholism by segmenting the GMV reduction at a specific region.

\section{Proposed method}

In this section, the proposed technique to detect GMV reduction in different regions of brain images is explained briefly. In this proposed technique, the collected brain images of both men and women are given to FCR model to segment the cerebrospinal fluid, gray matter and white matter. The major difference between FCM and FCR is modifying the Euclidean distance measure using additional parameter corresponding to the local linear model and hyperplane-shaped fuzzy membership function.

Consider $F=\left(X_{k}, y_{k}\right), k=1, \ldots, N$ is the set of input-output sample image pairs where $N$ refers to the number of samples, $X_{k}=\left[x_{1}, x_{2}, \ldots, x_{M}\right] \subset R^{m}$ is the $k^{\text {th }}$ input image vector, $M$ refers the number of fuzzy models, $y_{i}$ is the output of the $i^{t h}$ submodel, $y_{k}$ refers the $k^{t h}$ desired output for $X_{k}, \theta_{i}=$ $\left[b_{i}^{0}, b_{i}^{1}, \ldots, b_{i}^{M}\right], i=1, \ldots, c$ is the parameter vector of the $i^{t h}$ sub-model and $c$ is the number of fuzzy rules. This FCR model has a set of fuzzy rules, each defining a local input-output relation as follows:

Rule $i$ : IF $x_{1}$ is $A_{1}^{i}$ and $\ldots$ and $x_{M}$ is $A_{M}^{i}$, THEN

$$
y_{i}=\theta_{i}^{0}+\theta_{i}^{1} x_{k 1}+\cdots+\theta_{i}^{M} x_{k M}
$$

The output of this FCR model is given by:

$$
\hat{y}=\frac{\sum_{i=1}^{c} w_{i} y_{i}}{\sum_{i=1}^{c} w_{i}}
$$

In Eq. (21), $w_{i}$ is the fuzzy weight calculated as:

$$
w_{i}=\sum_{k=1}^{M} \mu_{A_{k}^{i}}\left(x_{k}\right)
$$

In Eq. (22), $\mu_{A_{k}^{i}}\left(x_{k}\right)$ is the fuzzy membership function of $x_{k}$ belonging to fuzzy set $A_{k}^{i}$. The fuzzy weight $w_{i}$ which is used for measuring the level of firing of the $i^{\text {th }}$ rule is computed as follows:

$$
w_{i}=\frac{\mu_{A_{i}}\left(x_{k}\right)}{\sum_{i=1}^{c} \mu_{A_{i}}\left(x_{k}\right)}
$$

Consider that the image pairs in $F$ are drawn from $c$ hyperplane-shaped clusters. The $i^{\text {th }}$ hyperplane-shaped cluster of $k^{\text {th }}$ input can be expressed as follows:

$$
\begin{aligned}
& y_{k}^{i}=b_{i}^{0}+b_{i}^{1} x_{k 1}+\cdots+b_{i}^{M} x_{k M}=\left[x_{k}, 1\right] \cdot \theta_{i}^{T}, \\
& i=1, \ldots, c
\end{aligned}
$$

The FCR clustering algorithm is based on the minimization of the sum of weighted distances between the training image dataset $F$ and the parameter vector $\theta_{i}$ in the $i^{\text {th }}$ hyperplane. The square of the distance $E_{i k}^{2}\left(\theta_{i}\right)$ are weighted with the hyperplane-shaped membership values $\mu_{i k}$ in the cost function (objective function) i.e., minimized by the FCR algorithm and described as follows:

$$
J(F ; U, \theta)=\sum_{k=1}^{N} \sum_{i=1}^{c}\left(\mu_{i k}^{m}\right) E_{i k}^{2}\left(\theta_{i}\right)
$$

Where the distance $E_{i k}\left(\theta_{i}\right)$ is defined as follows:

$$
E_{i k}\left(\theta_{i}\right)=\left|y_{k}-\left[x_{k}, 1\right] \cdot \theta_{i}^{T}\right|
$$

In the Eq. (25), $m \in(1, \infty)$ refers to the fuzzy weighted exponent and $\mu_{i k}$ refers to the membership degree of $x_{k}$ to the $i^{t h}$ hyperplane-shaped cluster. The fuzzy membership degree is measured by the distance between the input data and the hyperplaneshaped centers in the hyperplane-shaped membership function. In this FCRAD model, the fuzzy membership function is defined by the hyperplane-shaped Gaussian function as:

$$
\mu_{A_{i}}\left(x_{k}\right)=\exp \left(-\eta \cdot \frac{E_{i k}\left(\theta_{i}\right)}{\max \left\{E_{i k}\left(\theta_{i}\right), i=1, \ldots, c\right\}}\right)
$$

Here, $\eta \in[1,100]$ is a constant that tunes the fuzzy membership degree. The membership values $U=\left\{\mu_{i k}\right\}$ must satisfy the following constraints:

$$
\begin{aligned}
& \mu_{i k} \in[0,1], i=1,2, \ldots, c ; k=1,2, \ldots, N \\
& \sum_{i=1}^{c}\left(\mu_{i k}^{m}\right)=1, k=1,2, \ldots, N
\end{aligned}
$$




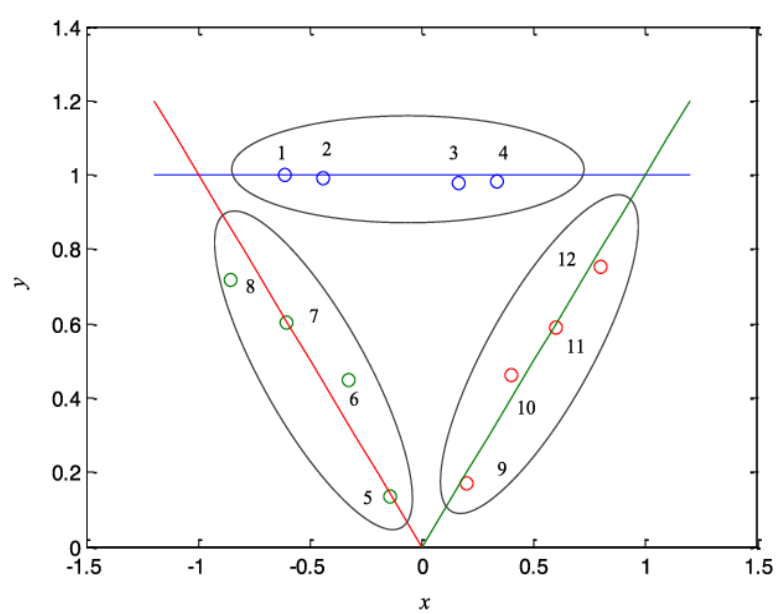

Figure.1 Example of hyperplane-shaped clustering

In Fig. 1, a dataset consists of 12 image pairs $\left(X_{k}, y_{k}\right)$ which are distributed around three lines is illustrated. It is noticeable that the input-output sample can be split properly by a hyperplane-shaped clustering algorithm such as FCRAD. The FCRAD is used for partitioning these image samples as well as obtaining the fuzzy weights $\left(w_{i}\right)$ and the membership degrees $\left(\mu_{i k}\right)$ by computing the fuzzy membership function $\left(\mu_{A_{i}}\left(x_{k}\right)\right)$ through (23) and (27), when the parameter of $\eta$ is set to 50 .

To achieve the FCR model, the following steps are performed:

For a given image $F$, assign $m>1$ and for specify the regression models (24), select an error measure i.e., distance (26) and a termination threshold $\epsilon>0$ and initialize $U^{(0)}$ randomly.

Repeat $\operatorname{for}(l=1,2, \ldots, \infty)$

Step 1: Compute the model parameters $\theta_{i}^{(l)}$ in Eq. (24) which globally reduce the cost function (25).

Step 2: Update membership function $U^{(l)}$ with $E_{i k}\left(\theta_{i}^{(l)}\right)$ to satisfy

$$
U_{i k}^{(l)}=\left\{\left[\sum_{k=1}^{c}\left(\frac{E_{i k}}{E_{j k}}\right)^{2 /(m-1)}\right]^{-1}, \quad \text { if } E_{i k}>0 \text { for } 1 \leq i \leq c,\right.
$$

Step 3: Stop until $\left\|U^{(l)}-U^{(l-1)}\right\| \leq \epsilon$, otherwise assign $l=l+1$ and return to Step 2 .

Further, a similarity factor is adopted to tackle the noise problem and obtain a number of homogeneous regions. The main difference in this proposed FCR model is the process of updating the membership function. The spatial information is obtained by the spatial function that defines the chance that pixel $x_{k}$ belongs to $i^{\text {th }}$ hyperplaneshaped cluster and computed as follows:

$$
h_{i k}=\sum_{i \in W_{i}} \mu_{i k}
$$

In the Eq. (31), $W_{i}$ is the local window centered on the pixel $x_{i}$ in the spatial domain. If the majority of its neighborhood belongs to the similar clusters, then the spatial function of a pixel for a cluster is large. The FCR iteration proceeds with the new membership function i.e., the spatial function is incorporated into the membership function as follows:

$$
\mu^{\prime}=\frac{\mu_{i k}^{p} h_{i k}^{q}}{\sum_{l=1}^{c} \mu_{l k}^{p} h_{l k}^{q}}
$$

Here, $p$ and $q$ are parameters which can control the respective significance of both functions. According to this, the membership function (30) is modified and the clustering results remain unchanged. This new spatial function can reduce the weighting of a noisy cluster by the labels of its neighboring pixels. As a result, misclassified pixels from noisy regions or spurious blobs are simply corrected. This iteration process is continued until the maximum difference between two clusters at two successive iterations is less than a termination threshold. After the convergence, defuzzification is applied for assigning each pixel to a specific cluster for which the membership is maximal. Thus, this clustering algorithm is used to segment the ROI i.e., the right posterior insula and the left thalamus from the entire brain image of men and women. For each region, the GMV value is determined and the variations in the GMV are computed as AET score. Finally, the mediation analyses are performed to examine whether the detected GMV variations of the ROI mediates the correlation between AET score and alcoholism.

\section{Algorithm:}

Input: Brain image dataset for both men and women

Output: Detected reduced GMV at left thalamus and posterior insula
1. Collect the training images;
2. for (each image sample)
3. \{
4. Randomly select the number of clusters;
5. Initialize the fuzziness factor;
6. $\operatorname{for}(i=1$ to max_iteration $)$
7. \{
8. $\quad$ Compute the distance $E_{i k}\left(\theta_{i}\right)$;
9. Compute the spatial function $h_{i k}$; 
10. Compute the membership values;

11. $\}$

12. if $\left(E_{i k}>0\right)$

13. \{

14. Update the membership values;

15. else

16. Compute the fuzzy cost function;

17. $\}$

18. Segment ROI;

19. Find the left thalamus region in men;

20. Find both left thalamus and posterior insula in women;

21. \}

22. Estimate AET score;

23. Perform mediation analyses;

24. Examine whether the detected GMV variations of the ROI mediates the correlation between AET score and alcoholism;

25. Observe the alcohol expectancy level;

26. End

\section{Experimental results}

In this section, the performance of the proposed FCR-based Alcoholism Detection (FCRAD) method is evaluated and compared with the existing GLMAD method [3], ANN \& K-NN [6], NB \& DT [7] and SVM [9] while applying for AD method by using MATLAB 2018a. In this experiment, the MIRIAD dataset is used which is examined from the Dementia Research Centre at the Institute of Neurology in London. In this dataset, totally 780 T1-weighted images are available in which 188 brain images are men with an alcoholic condition, 116 brain images are men with the healthy condition, 238 brain images are women with an alcoholic condition and remaining 138 brain images are women with the healthy condition. Among those, 400 brain images from each category are taken in this experiment. For training, 180 images are considered and the remaining 220 images are taken for testing. The comparison analysis is carried out in terms of precision, recall, f-measure and accuracy.

The experimental results of the detection of reduced GMV for GLMAD and FCRAD methods using both men and women under an alcoholic and healthy condition are shown in the following Table 1-4.

The mean \pm SD GMV of training images using FCRAD method are given in the following Table 5.

\subsection{Precision}

Precision is computed based on the prediction at true positive and false positive rates.

$$
\text { Precision }=\frac{\text { True Positive }(T P)}{\text { True Positive }(T P)+F \text { alse Positive }(F P)}
$$

Fig. 2 shows the comparison of proposed and existing alcoholism detection methods in terms of precision. From this analysis, it is observed that the overall precision of the proposed FCRAD method is $7.871 \%$ higher than all existing methods.

\subsection{Recall}

Recall is computed based on the yield prediction at true positive and false negative rates.

$$
\text { Recall }=\frac{T P}{T P+\text { False Negative }(F N)}
$$

The comparison of proposed and existing alcoholism detection methods in terms of recall is shown in Fig. 3. Through this graph, it is noticed that the overall recall of the proposed FCRAD method is $7.87 \%$ greater than the other existing methods.

\begin{tabular}{|c|c|c|c|}
\hline Methods & Input image & Segmented image & Detected image \\
\hline GLMAD & & & \\
\hline FCRAD & & & \\
\hline
\end{tabular}

Table 1. Detection of GMV reduction at left thalamus using men's brain image under alcoholic condition 
Table 2. Detection of GMV reduction at left thalamus using men's brain image under healthy condition

\begin{tabular}{|c|c|c|c|}
\hline Methods & Input image & Segmented image & Detected image \\
\hline GLMAD & & & \\
\hline FCRAD & & & \\
\hline
\end{tabular}

Table 3. Detection of GMV reduction at left thalamus \& posterior insula using women's brain image under alcoholic

\begin{tabular}{|c|c|c|c|}
\hline Methods & Input image & Segmented image & Detected image \\
\hline GLMAD & & & \\
\hline FCRAD & & & \\
\hline
\end{tabular}

Table 4. Detection of GMV reduction at left thalamus \& posterior insula using women's brain image under healthy

\begin{tabular}{|c|c|c|c|}
\hline Methods & Input image & Segmented image & Detected image \\
\hline GLMAD & & & \\
\hline FCRAD & & & \\
\hline
\end{tabular}


Table 5. Mean \pm SD GMV for brain images using FCRAD method

\begin{tabular}{|c|c|c|c|c|}
\hline No. of Images & $\begin{array}{l}\text { Men with Alcohol } \\
\text { Condition }\end{array}$ & $\begin{array}{l}\text { Men with Healthy } \\
\text { Condition }\end{array}$ & $\begin{array}{c}\text { Women with } \\
\text { Alcohol Condition }\end{array}$ & $\begin{array}{c}\text { Women with Healthy } \\
\text { Condition }\end{array}$ \\
\hline 50 & $\begin{array}{l}\text { Left Thalamus: } \\
363.78 \pm 65.07\end{array}$ & $\begin{array}{c}\text { Left Thalamus: } \\
417.46 \pm 59.03\end{array}$ & $\begin{array}{c}\text { Left Thalamus: } \\
\text { 341.35 } \pm 70.76 \text {; } \\
\text { Posterior Insula: } \\
344.56 \pm 68.78\end{array}$ & $\begin{array}{c}\text { Left Thalamus: } \\
\text { 383.07 } \pm 76.33 \text {; } \\
\text { Posterior Insula: } \\
393.94 \pm 64.51\end{array}$ \\
\hline 100 & $363.75 \pm 64.99$ & $417.42 \pm 59.02$ & $\begin{array}{l}341.31 \pm 70.74 \\
344.53 \pm 68.75\end{array}$ & $\begin{array}{l}383.06 \pm 76.31 \\
393.91 \pm 64.49\end{array}$ \\
\hline 150 & $363.71 \pm 65.02$ & $417.37 \pm 58.99$ & $\begin{array}{l}341.29 \pm 70.71 \\
344.49 \pm 68.72\end{array}$ & $\begin{array}{l}383.05 \pm 76.28 \\
393.88 \pm 64.46\end{array}$ \\
\hline 200 & $363.59 \pm 64.86$ & $417.31 \pm 58.96$ & $\begin{array}{c}341.26 \pm 70.68 \\
344.45 \pm 68.69\end{array}$ & $\begin{array}{l}383.04 \pm 76.25 \\
393.86 \pm 64.44\end{array}$ \\
\hline 250 & $363.47 \pm 64.93$ & $417.28 \pm 58.94$ & $\begin{array}{c}341.23 \pm 70.66 \\
344.41 \pm 68.64\end{array}$ & $\begin{array}{l}383.03 \pm 76.22 \\
393.82 \pm 64.41\end{array}$ \\
\hline
\end{tabular}

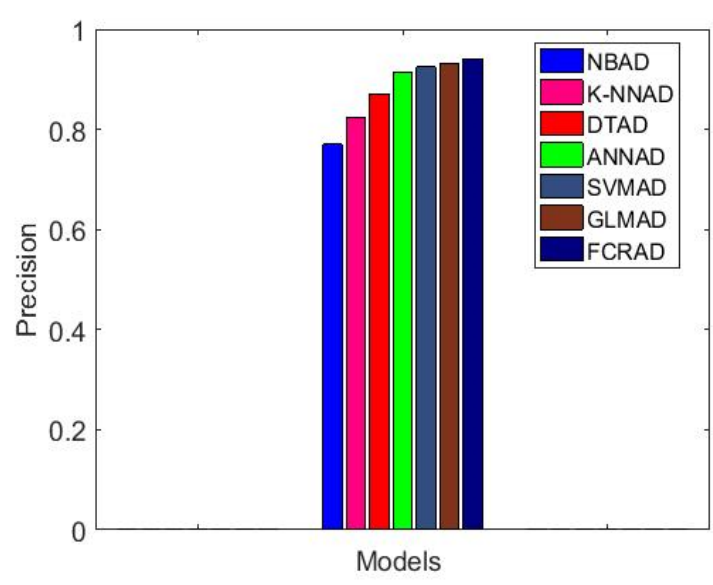

Figure.2 Comparison of precision

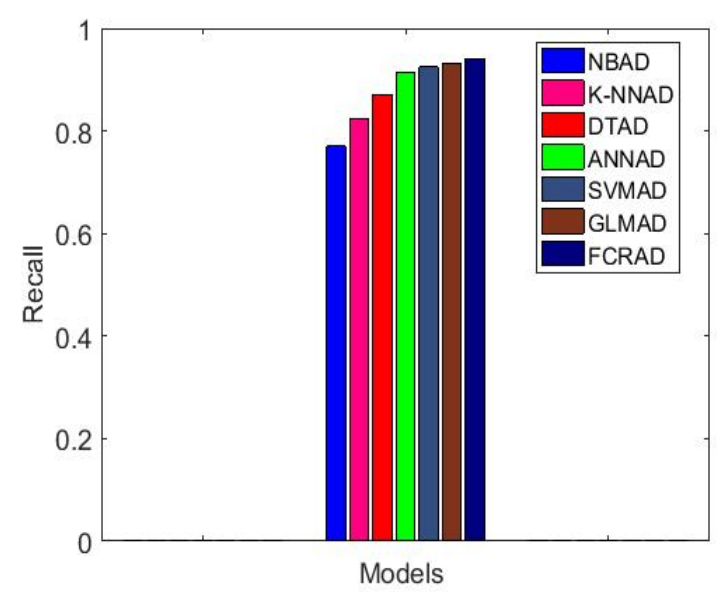

Figure.3 Comparison of recall

\subsection{F-measure}

F-measure is computed by using both precision and recall as follows:

$$
F-\text { measure }=2 \times\left(\frac{\text { precision } \times \text { recall }}{\text { precision }+ \text { recall }}\right)
$$

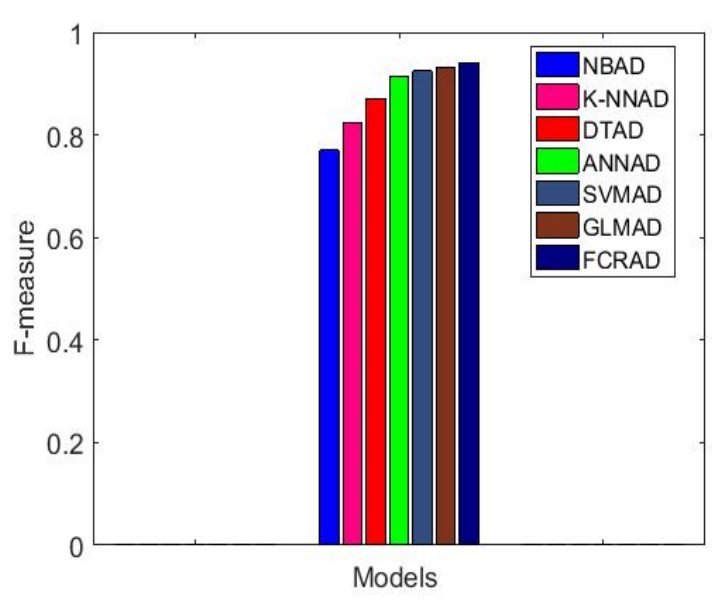

Figure.4 Comparison of f-measure

Fig. 4 illustrates the comparison of f-measure for both proposed and existing alcoholism detection methods. Through this analysis, it is observed that the overall f-measure of FCRAD method is $7.872 \%$ higher than all other methods.

\subsection{Accuracy}

Accuracy is defined as the proportion of both true positives and true negatives among the total number of cases examined.

$$
\text { Accuracy }=\frac{T P+T N}{T P+T N+F P+F N}
$$

The comparison of accuracy values for both proposed and existing alcoholism detection methods are shown in Fig. 5. From this analysis, it is observed that the overall accuracy of the proposed FCRAD method is $7.81 \%$ higher than all other existing methods.

Thus, it is proved that the proposed FCRAD method achieves better precision, recall, f-measure 


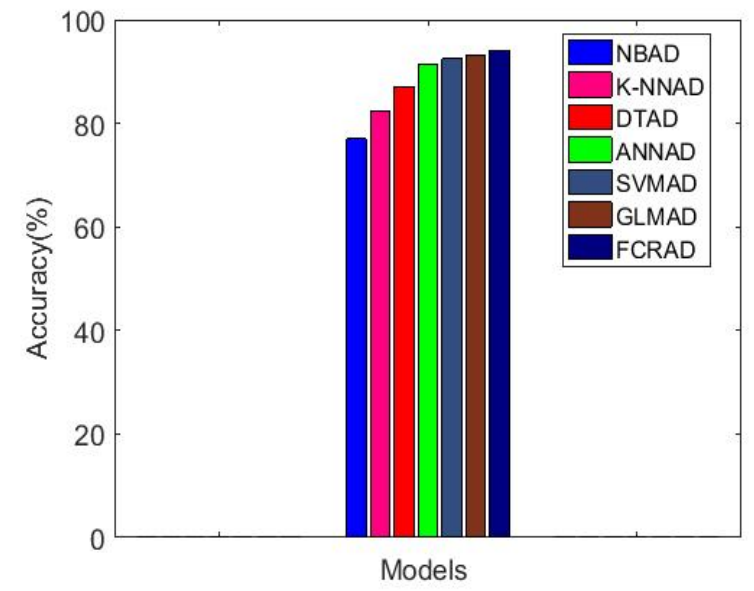

Figure.5 Comparison of accuracy

and accuracy than the other existing methods. The reason behind this improvement is that FCR clustering algorithm is hyper-planed and it can be suitable in either linear or nonlinear. Also, the spatial information is integrated in the FCR model to reduce the noise in images, while FCM algorithm is not appropriate for image segmentation due to lack of spatial information.

\section{Conclusion}

In this paper, GMV reduction is detected in different regions of the human brain based on the FCR algorithm. This technique supports both linearity and non-linearity of gray matter in the brain. In this segmentation algorithm, the effects of noise in the considered brain images are removed by incorporating the spatial information into the fuzzy membership function at each iteration. Once the segmentation is completed, GMV value at each region is estimated to compute the AET score. After that, mediation analysis is performed that mediates the reduced GMV to examine the correlation between AET score and alcoholism in both men and women. Finally, the experimental results prove that the proposed FCRAD method achieves an accuracy of $94.06 \%$ which is $7.81 \%$ higher than all other existing methods. Similarly, the precision, recall and f-measure values of FCRAD method is nearly $7.8 \%$ higher than GLMAD method. Thus, the proposed FCRAD method effectively predicts the alcoholic effects in both men and women. The future extension of this work includes the detection of GMV reduction at different regions of the brain along with white matter reduction.

\section{References}

[1] M. A. Enoch, and D. Goldman, "Problem drinking and alcoholism: diagnosis and treatment", American Family Physician, Vol. 65, No. 3, pp. 441-454, 2002.

[2] S. T. Mathew and M. Nachamai, "Clustering of brain MRI image using data mining algorithm", International Journal of Advanced Computational Engineering and Networking, Vol. 3, No. 4, pp. 3740, 2015.

[3] J. S. Ide, S. Zhornitsky, S. Hu, S. Zhang, J. H. Krystal, and R. L. Chiang-shan, "Sex differences in the interacting roles of impulsivity and positive alcohol expectancy in problem drinking: A structural brain imaging study", Neuroimage: Clinical, Vol. 14, pp. 750-759, 2017.

[4] S. Saha, A. K. Alok, and A. Ekbal, "Brain image segmentation using semi-supervised clustering", Expert Systems with Applications, Vol. 52, pp. 50-63, 2016.

[5] Y. Zhang, Z. Dong, L. Wu, and S. Wang, "A hybrid method for MRI brain image classification", Expert Systems with Applications, Vol. 38, No. 8, pp. 10049-10053, 2011.

[6] N. H. Rajini and R. Bhavani, "Classification of MRI brain images using k-nearest neighbor and artificial neural network", In: Proc. of IEEE 2011 International Conf. on Recent Trends in Information Technology, pp. 563-568, 2011.

[7] J. Naik and S. Patel, "Tumor detection and classification using decision tree in brain MRI", International Journal of Computer Science and Network Security, Vol. 14, No. 6, pp. 87, 2014.

[8] D. Sridhar and I. M. Krishna, "Brain tumor classification using discrete cosine transform and probabilistic neural network", In: Proc. of IEEE 2013 International Conf. on Signal Processing, Image Processing and Pattern Recognition, pp. 92-96, 2013.

[9] N. B. Bahadure, A. K. Ray, and H. P. Thethi, "Image analysis for MRI based brain tumor detection and feature extraction using biologically inspired BWT and SVM", International Journal of Biomedical Imaging, pp. 1-12, 2017.

[10] E. M. Ali, A. F. Seddik, and M. H. Haggag, "Using Data Mining Techniques for Children Brain Tumors Classification based on Magnetic Resonance Imaging", International Journal of Computer Applications, Vol. 131, No. 2, pp. 36-42, 2015. 